\title{
AN Evaluation METHOD OF RESEARCH ON WEARABLE WIRELESS BODY AREA NETWORK IN HEALTHCARE
}

\author{
Roberto Vito Rodrigues Filho ${ }^{1}$, Renato Freitas Bulcão Neto ${ }^{1}$, Bruno Oliveira \\ Silvestre ${ }^{1}$, Leandro Luís Galdino de Oliveira ${ }^{1}$, Rogerio Orlow de Oliveira ${ }^{2}$, and \\ Iwens Gervásio Sene Júnior ${ }^{1}$ \\ ${ }^{1}$ Instituto de Informática, Universidade Federal de Goiás, Goiânia - GO, Brazil \\ \{robertofilho, renato, brunoos, leandroluis, iwens\} einf.ufg.br \\ ${ }^{2}$ Faculdade de Medicina, Pontificia Universidade Católica de Goiás, Goiânia - GO, \\ Brazil \\ rogerioorlow@globo.com
}

\begin{abstract}
Wearable Wireless Body Area Networks (WWBANs) are an emergent technology enabling the gathering of real-time data, for instance, towards decision making by healthcare professionals in the case of remote monitoring of vital signs of patients. Recent research, however, does not describe in a straightforward way how hardware, software and wireless communication platforms fit together in order to fully support a $W W B A N$-based healthcare application. In this paper we propose a maturity analysis method of research on WWBANs in healthcare. The goal is to analyze whether a paper contemplates features that a research project on WWBAN in healthcare should have to apply it in a real world scenario. As a result of our method, features not addressed might indicate the lack of maturity of research papers, or even suggest open issues on that field.
\end{abstract}

\section{KEYWORDS}

WWBAN; WBSN; Healthcare; Maturity level;

\section{INTRODUCTION}

The more the worldwide population gets older, the bigger is the need for technologies capable to monitor and assist patients anytime anywhere so as to give them a more independent lifestyle. Even children or young people can also take advantage of the benefits of being monitored by healthcare professionals either in a properly instrumented place, e.g. a hospital, or at their own homes. In order to become this a reality, further improvements in wireless sensor-based communications are crucial. The remote monitoring of patients is one of the issues the Wearable Wireless Body Area Networks (WWBAN) has to tackle with, and it constitutes the main focus of this paper.

The WWBAN technology is a Wireless Sensor Network (WSN) sub-area that can be applied and presented as a better patient monitoring solutions than other technologies. That is because its ability to collect real-time information and easily match data against each other for better decision support. Most of the today's technologies are not able to collect and match different data as easily

DOI : 10.5121/ijcsit.2013.5105 
as the WWBAN, or send the data over the Internet or another broad network to a system to store, present, notify and assist healthcare professionals to make decisions.

The majority of the WWBANs projects according to [17] concentrate in issues related to heart diseases, asthma, emergency calls and stress monitoring. Though it is important to highlight the fact that WWBANs technology are not only applied in the healthcare field, it is also applied in military, entertaining, agriculture and even detection of earthquakes, but those last applications are not part of this paper.

One of the facts that motivated this work was not to find enough descriptions in many articles of how the hardware, software and communication patterns would fit together to compose a complete WWBAN application in the healthcare field. Sometimes articles do not fully detail the solution, e.g. poor description of communication protocols and how it is going to be used with commercial devices that do not have it natively implemented. This might be either because of the focus of the article which was more concerned in specific aspects of the solution itself or it might suggest the need of further investigation in order to point it out as an open issue that makes the research project not applicable in real world scenario.

This paper proposes a maturity analysis method of specific WWBANs articles applied in the healthcare area. A study of the articles of the area resulted in a set of aspects relevant to detect its applicability in a real world scenario. These aspects were taken into account to compose this method. As a contribution, the method proposed points out specific characteristics that may present open issues in the WWBAN area applied to the healthcare field.

This paper is organized as follows the Sections 2, 3 and 4 describes the hardware and software platforms and the communications standards respectively, as fundamental characteristics to define the method proposed in Section 5, which describes articles selected in order to demonstrate the maturity method described in Section 6.

\section{HARdWARe Platforms}

There is a vast range of hardware platforms used to compose a WWBAN application, specially applied to healthcare. In this article we are going to be presenting the hardware platforms used to compose the research projects that are going to be described later in this paper.

Firstly it is important to point out some hardware characteristics that a healthcare targeting application needs. The healthcare application in general, considering it is going to be collecting data from the human body, should not hinder the patient's movements and be as comfortable as possible, in other words, it should be light-weighted, small and noninvasive. On a side note, since our target is the wearable devices, the implantable sensors will not be considered.

Being small and light-weighted is a challenge, especially if considered the size of the device being mostly the size of its battery. The battery size has a direct relation to the amount of energy it can store and thus since sensors applications usually requires a fair amount of energy it will automatically require a fair size of battery. In other words the challenge lies in developing small and lightweight batteries that does not compromise the life-time of the applications.

The hardware should also be wearable and should be strategically attached in the human body, since it is going to be in direct contact with possible injured patients with open skin and blood the devices should also be easily washable and sterilizable. The manufacturer has also to be careful with the material in which the hardware will be fabricated since it will be in contact with the skin, allergies and irritations must be avoided. 


\subsection{Motes}

The concept of a mote comes from the Wireless Sensor Network (WSN) and it is basically a device composed by a memory, a processing unit and energy supply on which the sensors and actuators are attached.

The Telos and Mica mote platforms are widely used in the WSN applications and after getting healthcare sensors adapted to them [4] became widely used in the healthcare field. The Telos platform is basically composed by a MPS430 microcontroller, a 2 AA battery pack and CC2420 radio interface with $2.4 \mathrm{GHz}$ band and reaches 50 meter indoors and 128 meters outdoors. It also has a USB for programming, and comes with humidity, temperature and light sensors attached to it. The Mica platform is slightly different; it is composed by an Atmega 128L microcontroller, a 2 AA battery pack and a CC1000 radio interface with 868-870; 902-928 Mhz and reaches 30 meter indoors.

The characteristics of the Mica and Telos motes are not quite suitable for healthcare applications, as it was stated in [4], and some other platforms were proposed in order to be specifically applied to healthcare environments, which is the case of the Pluto platform described in [4] and the Shimmer platform described in [11]. Pluto is based on Telos hardware platform. It also has the MSP430 microcontroller and the radio interface CC2420 but its antenna is slightly different and instead of a pack of 2 AA batteries it is supplied by a tiny rechargeable $120 \mathrm{mAh}$ lithium polymer battery, it was proven to function for nearly 5 hours. It has a Mini-B USB port for programming and recharge and it is compatible with the TinyOS.

The Shimmer platform on the other hand is a commercially available wearable hardware platform also compatible with TinyOS, and its hardware is composed by the MSP430 microcontroller, the CC2420 radio interface and a rechargeable Li-ion battery.

\subsection{Biosensors}

There are a lot of physiological signals a sensor can collect from the human body and the decision of which signal to collect depends on the application's requirements. In the healthcare field there are certain important signals to determine someone's health state, those signals are the ones that are most used in this kind of applications, those are: ECG, Pulse Oximeter and Blood Pressure. Depending on the application there are going to be other signals complementing those or there are going to be different signals, for instance, in an application that aims to detect patients' falls should have accelerometers and gyroscopes instead.

Table 1. List of signals and the sensors capable of collecting them.

\begin{tabular}{|l|l|}
\hline Signals & Sensors \\
\hline Heart Electrical Signals & Electrocardiogram (ECG) \\
\hline Muscles Electrical Activity & Electromyogram (EMG) \\
\hline Blood Oxygenation & Pulse Oximeter \\
\hline Blood Pressure & Blood Pressure Cuff \\
\hline Body Temperature & Thermometers \\
\hline Motion & Accelerometers and Gyroscopes \\
\hline Skin Conductivity & Galvanic \\
\hline Heart Electrical Signals & Electrocardiogram (ECG) \\
\hline
\end{tabular}


The sensors and signals listed in Table 1 are the most used sensors in the healthcare projects. And some of them, such as ECG, Pulse Oximeter and the Blood Pressure are indispensable to monitoring the vital signs of the patients [5].

Also, according to [5] the pulse oximeter has limited accuracy readings with methemoglobin, carboxyhemoglobin, nail polish, nail fungus, fluorescent light and motion and thus must be carefully used in WWBANs applications.

Other sensors usually attached to the motes are the GPS and an electronic tag used for triage purposes but they are not classified as biosensors.

\section{Software Platforms}

The WWBAN technology when applied to the healthcare field requires basically three software layers; the one used in the sensors motes, the one used in the personal server (PS) and the one that composes the server-side of the application. There are a great variety of technologies that compose the server-side, since it basically addresses development of multipurpose systems, in the healthcare case generally it basically displays data collected from the patients, and send alert notifications as needed.

The second layer, the one that lies on the PS which commonly has as a device a cell phone or a PDA, is composed of commercially available software for those specific devices. The software that composes this layer as well as the server-side is not the focus of this paper, and the problems of these layers concerning communication, security, healthcare data handling can be addressed with the commercially technologies and standards available.

This paper focuses on the sensor motes and there are plenty of work on operating systems, frameworks and middleware platforms for embedded devices that compose a WSN. Though many of these works do not have stable versions of software or was discontinued.

Besides those, one project that is widely used and has an active community in the operating system area is the TinyOS. The TinyOS is an operational system vastly used in the general WSN field due to its design characteristics. It has a component-based programming model and uses $\mathrm{NesC}$ as a programming language, a dialect of $\mathrm{C}$, it is not an operational system in the traditional sense, it is more of a framework and a set of components to develop embedded systems applications as mentioned and described in [9].

Another project that has an available version is the software platform CodeBlue. The CodeBlue platform addresses the issues in the hardware (as described in the last section, with the Pluto hardware platform) and the software; most importantly it is focused in the healthcare field. The CodeBlue software platform runs on top of the TinyOS, and it is described in [4] as a protocol and middleware framework for medical applications. In other words the software platform comes to make the hardware platform developed to healthcare applications functional, and adds protocols and services such as routing protocols and query services that addresses the healthcare applications challenges and help implementing applications in the healthcare field.

As mentioned before there are not only this two software platforms, there a many projects that develop software specifically to their hardware platforms, but the TinyOS and CodeBlue are the most commonly used in the WWBAN applied to the healthcare field and they are presented in the great majority of the research projects that will be described in a later section. 


\section{Wireless Communication Standards}

In this section it is going to be presented some important and widely used communication standards in the WWBAN technology and most importantly the problems when they are used to compose WWBAN-based applications. Some of the issues presented in this section are also described in [15].

The ZigBee/IEEE 802.15.4 standard communication is widely used in this kind of close range network, e.g. [19], mostly due to its low energy consumption and cost, this standard characteristic is best described in its documentation [13]. One of the issues is that some environments which use WWBAN technology might also have a WLAN, in [14] it argues that the WLAN might interfere with ZigBee. Another issue that the WWBAN applied in the healthcare field suffers is with the highly variable path loss near the human body, in [12] mentions a project that uses the Telos hardware platform which is composed of a IEEE 802.15.4 radio attached to a patient's chest that was not able to communicate with another Telos located at the patient's back.

The Bluetooth is also a well known communication standard and are implemented in many commercial devices, such as laptops, cell phones and tablets, and thus it is also widely used. It was designed to consume low power energy but apparently would consume too much energy to connect mobile terminals wirelessly with small devices that has very little energy supply, with that proposal it was designed the Bluetooth Low Energy technology, which can be suitable to compose the hardware of a WWBAN, specially in the healthcare field since the mote should be very small. The Bluetooth is described in detail in [16].

Table 2. Communication Protocols extracted from [10].

\begin{tabular}{|l|l|l|l|l|}
\hline Standards & Range & Data Rate & Cost per Chip & Frequency \\
\hline ZigBee & $10-75(\mathrm{~m})$ & $20 \mathrm{kbps} /$ & $\$ 2.00$ & $868 \mathrm{MHz} /$ \\
& & $40 \mathrm{kbps} /$ & & $915 \mathrm{MHz} /$ \\
& & $250 \mathrm{kbps}$ & & $2.4 \mathrm{GHz}$ \\
\hline BlueTooth & $10-100(\mathrm{~m})$ & $1-3 \mathrm{Mbps}$ & $\$ 3.00$ & $2.4 \mathrm{GHz}$ \\
\hline
\end{tabular}

The Table 2 illustrates the two wireless communication protocols that were described in this section along with the range they reach, data rate, cost per chip and their frequency. These are the most used standards in WSN technology.

This paper does not intend to propose solutions to the problems listed in this section, since the majority of this problems lies in the IEEE 802.15.4 which was the most used standards in the research projects that will be described in the next session, but we believe the solution is in the development and investment on new technologies standards such as the Bluetooth Low Energy and the study and development of new communication protocols.

\section{RESEARCh PROJECTS ON WWBANS}

In this section it is presented some WWBANs research projects used to demonstrate the evaluation method in the Section 6. The research projects will be described along with some of their features such as the hardware platforms, software platforms, communication patterns and biosensors. It is important to mention that these projects were chosen due to the following criteria: i) being relevant; ii) having wireless communication; iii) having wearable sensors; iv) being applied to the healthcare field. 
The relevance was a criterion based on the Google Scholar which takes into account the author's ranking, the number of references linked to it, the ranking of the publication that journal appears, among others. The other reason why Google Scholar was chosen was because it indexes a large number of articles from different search bases.

The wireless communications was a criterion to choose the research projects because it is an important requirement for healthcare applications, since the wires can cause skin irritation and specially hinder the patient's movements. The best case scenario should be the absolute absence of wires and cords, but the technology has not evolved enough thus far. However this paper is more concerned in the projects which the communication from the personal server (cell phones, PDA, etc...) to a computer or access point is wireless to allow the patient to move freely in an environment, and to make it possible to collect data from patients remotely.

The projects described in this paper makes usage of nonintrusive sensors, specifically wearable sensors, it is known that intrusive sensors such as implantable sensors has some challenges, for instance, due to the skin resistance there are frequently loss packages in the data communication, another challenge is the energy consumption, due to the fact that the sensor is implanted under the patient's skin making it more complicated to recharge. Those problems and challenges are out of this paper's scope, thus the projects listed here will only be the ones that use wearable nonintrusive sensors.

It is well known that WWBAN technology is applied in a great variety of fields; it can be used in the military attaching wearable sensors to a soldier or fire-fighter so it can measure the levels of toxic substances in the environment and warn the user of the risks. It also can be used to train golf athletes to perfect their swing, for example, attaching motion sensors to them and run algorithms to detect eventual mistakes. But all of these other applications of the technology are out of the scope of this paper, since it focuses only on the healthcare field.

One of the research projects was developed in the Division of Engineering and Applied Sciences of the Harvard University. It was developed the software and hardware platform for medical care. Attempting to fill out the gap between the need of medical care and the existing technology in WSN it was developed the CodeBlue [4]. The CodeBlue platform is composed by the hardware and software platforms that addresses the healthcare field, the hardware platforms were built on top of the widely used MicaZ, Mica2 and Telos motes, it was created medical sensors such as ECG, pulse oximeter and a specialized motion-analysis sensor board. The software platform is the CodeBlue framework built on top of the TinyOS [9] and provides protocols for device discovery, publish/subscribe multihop routing, and a simple query interface for care professionals access one or a group of patients.

The Real-Time Wireless Physiological Monitoring System (RTWPMS) described in [2] is a research project that monitors aged patients on nursing centers. It collects real-time heart rate, pressure and body temperature. The patient is monitored and gets his data stored, so he can be real-time monitored or get his data analyzed latter to detect changes in his health status. The hardware platform of the system is composed mainly by four parts; a mobile physiological examination device(s); a wireless base station(s); a voice/data exchange device; and a network management center, the hardware platform was developed specifically to this application. The software platform was developed based on a modular approach in an object-oriented way to make it easy to update, modify and maintain.

The Wireless sensor networks for personal health monitoring (WPHM) [3] is an article that describes the utilization of BAN technology in a healthcare application. It points out some architecture concepts describing generally three layer architecture among others WWBANs 
concepts. The WPHM [3] also describes in more detail a prototype that aims to cover the challenges described in the article, such as small size, low power consumption, secure communication, and interoperability. It uses the ActiS hardware platform with custom sensor platform, it monitors the heart rate as well as the position of the upper body trunk and two motion sensors attached to the patient's ankle to monitor his activity. It makes it possible to correlate the steps and body movements with the patient's heart rate to determine medical conditions. The Actis hardware platform is made of two custom boards designed specifically for health monitoring applications attached to the commercial Telos platform. One of the boards is the Intelligent Signal Processing Module (ISPM) that extends the Telos features and adds to it two perpendicular dual axis accelerometers (Analog Devices ADXL202), a bioamplifier with signal conditioning circuit, and a microcontroller MSP430F1232. The other board is the Intelligent Activity Sensor (IAS) which is a version of the ISPM with only accelerometer sensors and signal conditioning. The software platform used to coordinate the motes as well as the sensor boards is the TinyOS platform.

The Advanced Health and Disaster Aid Network (AID-N) described in [5] is another project that applies the WWBAN technology in the healthcare field. It utilizes the technology to feed data to the Advanced Health and Disaster Aid Network of patients and to make the triage and better send them to the hospital. It uses an ECG, blood pressure and pulse oximeter as well as a triage tag to continuously monitor the vital signs of a patient and deliver pertinent information to first responder. The triage tag is utilized to disseminate data from the incident site to some key members of the distributed emergency response community which makes it faster and triple the number of patients triaged compared to the paper system. It uses a MicaZ or TmoteSky platform and an ETag, ECG, blood pressure, and pulse oximetry sensors attached to it and for the software platform it used the CodeBlue framework on top of the TinyOS.

Another WWBAN system is the CareNet described in [6], the project basically provides a remote healthcare system that provides data privacy, scalability, extensibility and integration with webbased patient portal between the patient at home and the care givers, the system monitors the physical activity of the patient remotely using Telos motes equipped with accelerometers and gyroscopes to detect movements and fall detection. The software platform was developed on top of the TinyOS.

In the healthcare field the monitoring of patients, usually older patients, in an assisted-living community is a common scenario. In this scenario there are a lot of challenges to be addressed and solved, in [7] it is described a Wireless Sensor Networks for Assisted-Living and Residential Monitoring, the ALARM-NET project, that addresses those challenges and propose a system to monitor those patients. The system's hardware platform is composed by MicaZ motes with infrared motion, dust sensors, temperature, light, pulse, and blood oxygenation sensors and it is developed using TinyOS software platform.

The problem of monitoring older patient's body posture for falling detection has been solved in different ways; one of them is described in the Adaptive Body Posture Analysis Using Collaborative Multi-Sensors for Elderly Falling Detection (ABPA) in [8]. It uses sensors attached to some parts of the patient body to calculate the body posture and gets high accuracy, in this project it is used tri-axial G-sensor on the neck the neck, waist, left wrist, right wrist, left thigh, and right thigh, and the software platform used was Triax Reloaded and Matlab.

\section{Maturity LeVel}

The maturity level method proposed may evaluate articles and determine whether or not it can be applied in a real world scenario. In this section we describe how the method is applied to analyze 
the articles listed in previous section as an example of the method's applicability and to point out the results obtained. It is important to remark that this method is manually conducted. A series of research projects should be read and the set of characteristics pointed by this method must be identified in them.

The research projects are evaluated, as mentioned, according to a set of characteristics described in [10]. As a result, articles ranked in a high position satisfy more of those characteristics and thus are mature enough to be used in real world scenario. Those characteristics were elected to be essential for the research project to be used in a real world scenario and aims to supply key factors from the perspective of the healthcare professional, the patient and the developer/manufacturer. Each characteristic is more important to one perspective than other but they are all essential for the maturity level of the research project in general.

According to [10] the Table 3, a table which was extracted and extended from [10], points out the characteristics and its weight. The weight was calculated based on the patient's, physician's and manufacturer's perspective.

The characteristic's weight can assume values from 3 to 1 , being 3 important and 1 not so important or impacting from the evaluators' perspective. The weights given for each characteristic and from each perspective (patient, physician and manufacturer) are described in more detail in [10]. The Table 3 only shows the average weight between each perspective and thus gives us generally how crucial and impacting the characteristic is, in other words it tells us if the characteristic is important to all perspectives or just one or two. The average itself is calculated summing up the weights from each perspective and dividing this value by three, so if the weight is 2.3 it means that from two perspectives it got weight 2 and from one it got weight 3 . One can conclude that the higher the weight average gets the more impacting the characteristic is because it impacts more perspectives and thus it is crucial when applying the research project in a real world scenario. However one should be careful to jump into the conclusion that if the weight average is low the characteristic is not impacting and thus it is not so important when applying the research project in a real world scenario, the last piece is not true since it only means it does not impact all perspectives but can impact the appliance of the research project in the real world, specially if the characteristic affects the functioning aspects of the solution.

Another important observation is that most of the characteristics is crucial and impacting since it got the minimum average weight of 1.7, in other words it got weight 2 in two perspectives and weight 1 in only one, so the minimum average weight characteristic has medium impact from the perspective of two different evaluators and a low impact in only one. The characteristics and its weights are shown bellow. 
International Journal of Computer Science \& Information Technology (IJCSIT) Vol 5, No 1, February 2013

Table 3. The characteristics' list.

\begin{tabular}{|c|c|c|}
\hline Characteristics & Description & Weight Average \\
\hline Wearability (1) & The system must have low weight and size & 2 \\
\hline $\begin{array}{l}\text { Appropriate } \\
\text { placement on } \\
\text { the body (2) }\end{array}$ & $\begin{array}{l}\text { The system has to be unobtrusive and comfortable, } \\
\text { in order to not interfere with the user's movements } \\
\text { and daily activities. }\end{array}$ & 2 \\
\hline $\begin{array}{l}\text { Aesthetic issues } \\
\text { (3) }\end{array}$ & $\begin{array}{l}\text { The system should not severely affect user's } \\
\text { appearance. }\end{array}$ & 2 \\
\hline $\begin{array}{l}\text { Data encryption } \\
\text { and security (4) }\end{array}$ & $\begin{array}{l}\text { Encrypted transmission of measured signals and } \\
\text { authentication requirement for private data access. }\end{array}$ & 3 \\
\hline $\begin{array}{l}\text { Operational } \\
\text { lifetime (5) }\end{array}$ & $\begin{array}{l}\text { Ultra low power consumption for long-term, } \\
\text { maintenance-free health monitoring. }\end{array}$ & 2.3 \\
\hline $\begin{array}{l}\text { Real-application } \\
(6)\end{array}$ & $\begin{array}{l}\text { The developed system is applicable (and useful) to } \\
\text { real-life scenarios/health conditions. }\end{array}$ & 3 \\
\hline $\begin{array}{l}\text { Real-time } \\
\text { application (7) }\end{array}$ & $\begin{array}{l}\text { The wearable system produces results, e.g. display of } \\
\text { measurements, alerts, diagnosis etc, in (or near) real- } \\
\text { time. }\end{array}$ & 2.3 \\
\hline $\begin{array}{l}\text { Computational } \\
\text { and storage } \\
\text { requirements (8) }\end{array}$ & $\begin{array}{l}\text { The computation and the storage required or utilized } \\
\text { by the system to achieve desirable results. }\end{array}$ & 1.7 \\
\hline Easy of use (9) & $\begin{array}{l}\text { The system incorporates a friendly, easy-to-use and } \\
\text { easy-to-learn user interface. }\end{array}$ & 2 \\
\hline $\begin{array}{l}\text { Performance } \\
\text { and test in real } \\
\text { cases }(10)\end{array}$ & $\begin{array}{l}\text { Sufficient results and performance statistics are } \\
\text { provided to verify the system's functionality in real } \\
\text { cases. }\end{array}$ & 3 \\
\hline Reliability (11) & The system produces reliable and accurate results. & 3 \\
\hline Cost (12) & $\begin{array}{l}\text { The amount of money required to produce and } \\
\text { purchase the proposed wearable system. }\end{array}$ & 2.3 \\
\hline $\begin{array}{l}\text { Interference } \\
\text { Robustness (13) }\end{array}$ & $\begin{array}{l}\text { Availability and reliability of wirelessly transmitted } \\
\text { physiological measurements. }\end{array}$ & 2.3 \\
\hline $\begin{array}{l}\text { Fault Tolerant } \\
\text { (14) }\end{array}$ & $\begin{array}{l}\text { The system produces reliable results under any } \\
\text { circumstances, such as various kinds of patient's } \\
\text { movements. }\end{array}$ & 3 \\
\hline Scalability (15) & $\begin{array}{l}\text { Potentially of upgrading, enhancing and easily } \\
\text { incorporating additional components to the } \\
\text { developed system. }\end{array}$ & 2 \\
\hline $\begin{array}{l}\text { Decision } \\
\text { support (16) }\end{array}$ & $\begin{array}{l}\text { The implemented system includes some type of } \\
\text { diagnosis/decision mechanism or an } \\
\text { algorithm/pattern recognition system for context } \\
\text { aware sensing of parameters. }\end{array}$ & 2.3 \\
\hline
\end{tabular}

It will be presented the results after the process of evaluation. For each article it was verified if it contemplates the listed characteristics. So for each characteristic in each research project received a score that represents how well described, or if described at all, the characteristic was in the article. The score vary from 0 to 2 . Being 0 very little or none detail or does not apply, 1 it is described but lacks some details, and 2 the characteristic was described very detailed and was well used in the research project. 
In a side note it is known that the research projects that are being evaluated have more than one article published and some are still being developed. But even so the following results were based in only one article of each project. It has to be clear that it was considered only the concepts shown in the project's article as to exemplify the method being proposed, even though it is known that a full analysis must consider all the articles produced in the research project.

Taking the characteristics listed above and verifying if each one of them is considered in each article is a step-by-step way to evaluate WWBANs research projects applied to healthcare field. The resulting analyses led to features that are not receiving attention by the articles. The reason why a characteristic is being targeted in an article and not in others can suffer from a great range of reasons, but the intent is to firstly identify the characteristics most presented and the ones that are not. So the characteristics that are not described in the majority of the articles become a topic of interest which can be identified as open issues in a future work that would analyze the literature to confirm it.

It is important to point out that the articles that do not take into account some of the characteristics specifically because they are not crucial parts of the solution (e.g. adequate hardware platforms) will get a low level of maturity. That is because some characteristics can impact directly the way the application would work, for instance, the solution might use an algorithm that demands energy that an adequate hardware platform could not provide, and by not considered these aspects the WWBAN-based application would not work in a real world scenario. Analyzing the research projects we arrive in the resulting graphic that gives an overview of the maturity levels, it is illustrated in the Figure 1.

\section{Maturity Level Overview}

\section{Projects Maturity Level}

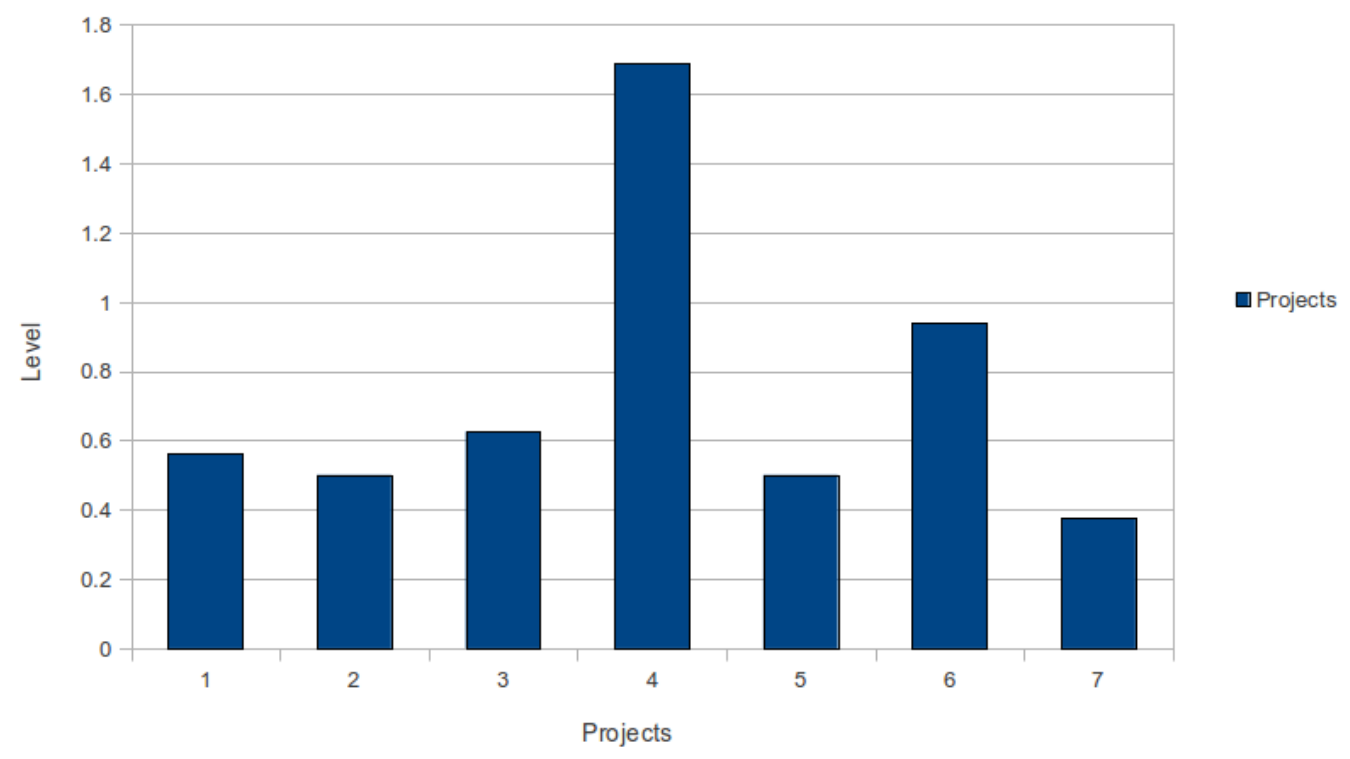

Figure 1. 1 - RTWPMS; 2 - WPHM; 3 - CodeBlue; 4 - AID-N; 5 - CareNet; 6 - ALARM-NET; 7 ABPA. Resulting Graphic: Maturity Level Overview.

The result plotted in the graphic shows what articles better consider the characteristics listed. It has to be noted that some articles do not consider some set of characteristics due to its focus. The ABPA is an example. It has a very good approach to the falling detecting problem giving a 
method very accurate to identify falls and it basically considers only that. On the other hand it does not consider other fundamental characteristic that must be considered for the solution to be applied in real world scenarios and thus had the worse average score.

The graphic points out the project number four, the research project AID-N, as the one that has the majority of the characteristics better described in its article. The AID-N article analyzed covers most of the characteristics in a detailed way, it is a very complete article and gives a very detailed description of the project as well as their concerns on the healthcare area's problems, such as scalability, wearability, fault tolerance, cost, among others that compose the characteristic's list.

The characteristics that were not detailed described were: data encryption and security, interference robustness and decision support. Since the AID-N applies the platforms developed in the CodeBlue project it states that even though security is very important in the healthcare field to keep the privacy of the patients data it is a conflicting requirement with the scalability of the system, since the security generates overhead in the network's communication, so in this case it was invested in the scalability of the project over the security.

On the other hand after analyzing all the projects selected we can see each of the characteristics were better detailed in the projects. From that perspective the resulting graphic illustrated in the Figure 2 shows for each characteristic on the y-axis how much each of them was described in the articles.

\section{Characteristics in the Projects (Overview)}

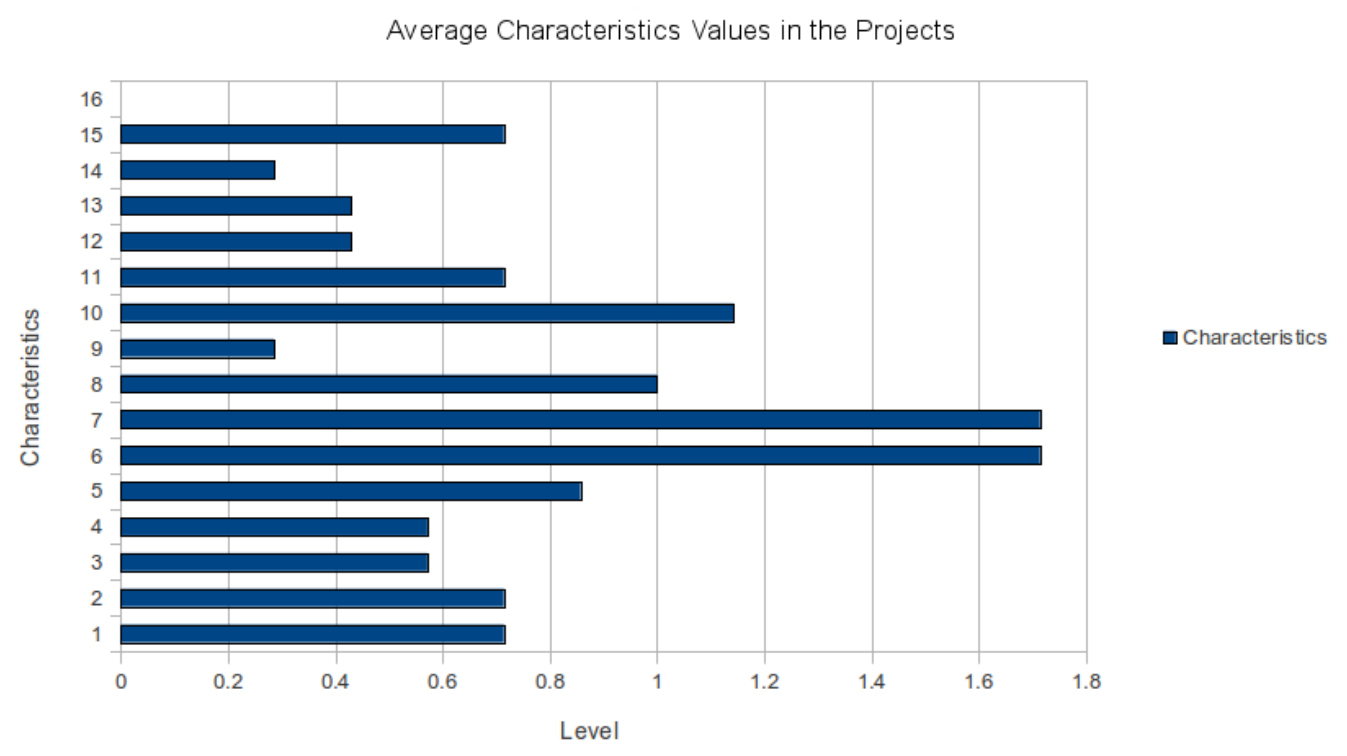

Figure 2. Resulting Graphic: Characteristics in the Articles.

This resulting graphic is very important considering it shows which characteristics were better described in the articles and which ones were not.

The Decision Support characteristic, for example, was not detailed in any project analyzed according to the resulting graphic. One can misinterpret this information since all of those projects are support systems, so generally they provide tools and information to the healthcare 
professional that supports decisions making and thus they support decision. But that characteristic as far as this analysis was conducted considers if elaborated computational techniques were applied to assist in the decision making by the healthcare professionals.

Another issue that can be pointed out is the lack of healthcare professionals in the research project, some of the articles describes the presence of healthcare professionals in the project but not all, so problems such as easy of usage, wearability concerns and cost sometimes are not well described. And since it is a system that provides tools and real-time information to healthcare professionals, their presence in the project is essential to feed the project with the application field's concerns and for their evaluation.

The majority of hardware platforms used is the Berkley motes with adapted healthcare sensors, the most commons were the Telos and Micaz which is a great commercial sensor platform for WSN in general, but as mentioned in [4] they are not quite adequate to use as wearable sensors due to its size and weight.

The security aspects of the healthcare application seems to appear in many projects due to the patient's data privacy, but most of the articles fail to detail techniques, algorithms or software/hardware platforms that addresses the security aspects, especially since a secure system can cost other important requirements of healthcare applications that can be compromised such as scalability [4].

The reliability and fault tolerance characteristics which are very important to a healthcare system in the resulting graphics of the characteristics did not get a satisfying level of details in the articles. It is kind of tricky to provide fault tolerance mechanisms to a healthcare sensor application since classical approaches such as redundancy might not be possible by aesthetic issues, hinder patient's movements and cause discomfort.

Another topic is the standards in the WWBAN technology applied in the healthcare field; the standards can provide system interoperability in all levels of a healthcare system. They are not presented in any article of the projects analyzed.

The usage of standards in a WWBAN application facilitates the integration of the various software and hardware platforms. The projects listed in this paper do not mention the usage of any healthcare standards but a lot of attempts to make interoperability possible in the healthcare application technologies were made in the literature. The Health Level Seven (HL7) and the ISO/IEEE 11073-10471 are examples of healthcare standards for telehealth and bedside devices.

The HL7 defines and standardize the integration between healthcare systems defining languages, structure, data types, data sending and packaging to integrate systems. It also defines management, delivery and evaluation of healthcare services. The HL7 standard is recognized as the most commonly used in the world. The ISO/IEEE 11073-10471 on the other hand establishes and defines the communication between telehealth independent devices and computers (such as cell phones, personal computers, personal health appliances) to provide interoperability plug-andplay. Those standards differ in the way they address certain needs. The ISO/IEEE 11073-10471 addresses the need of an openly defined and independent standard to control the exchange of the information between the personal devices and the computers. The HL7 is more complete in the sense it addresses the need to integrate, exchange, share, and retrieve electronic health information. 


\section{Conclusions/OutlooK}

WWBANs are a promising technology that addresses a great variety of challenges presented nowadays in different areas. One of the areas that can greatly benefit from this technology is healthcare. Although this technology is very promising in the medical field some research projects do not describe in a straightforward way how hardware, software and wireless communication platforms fit together in order to fully support a WWBAN-based healthcare application. Thus the maturity analysis method proposed assists researchers to spot lacking characteristics in articles that are important for a research project when applied in a real world scenario.

Characteristics such as inappropriate hardware platform, software platforms not covering all the healthcare requirements and communication standards presenting limitations when applied in healthcare environment were commonly found among the selected articles analyzed with our method. Specifically using light-weighted, washable, wearable, unobtrusive and sterilizable motes in regards to hardware and data security and elaborated computational techniques for decision support in regards to software, another issue that would greatly contribute to this type of research is having healthcare professionals working actively in the project.

Research projects that do not consider the test of a solution according to those characteristics received for their articles a low level of maturity because their solutions might be invalid in a real world scenario and that is the main goal of the method. Some of those lacking characteristics that were common among all the articles analyzed might suggest an open issue, therefore further research has to be conducted to confirm it.

\section{ACKNOWLEDGEMENTS}

The authors would like to thank the reviewers of the paper for their valuable comments. We also thank the CNPq funding agency for the grants n. 560269/2010-2 and 481402/2011-0.

\section{REFERENCES}

[1] Gao, Tia.; Greenspan, Dan; Welsh, Matt; Juang, Radford; Alm, Alex; C.D. Vital Signs Monitoring and Patient Tracking Over Wireless Network. In Proceedings of the 27th Annual International Conference of the IEEE EMBS 2005.

[2] Bor-Shing Lin; Bor-Shyh Lin; Nai-Kuan Chou; Fok-Ching Chong; Sao-Jie Chen ; C.D. Real-time Wireless Physiological Monitoring System (RTWPMS). IEEE Transactions on Information Technology in Biomedicine, Vol. 10, N. 4, 2006.

[3] Milenkovic, Aleksandar ; Otto, Chris ; Jovanov , Emil; C.D. Wireless Sensor Networks for Personal Health Monitoring: Issues and an Implementation. Computer Communications 292006

[4] Shnayder, Victor; Chen , Bor-rong; Lorincz, Konrad; Fulford-Jones, Thaddeus R. F; Welsh , Matt C.D. Sensor Networks For Medical Care. Technical Report TR-08-05, Division of Engineering and Applied Sciences, Harvard University, 2005.

[5] Tia Gao; Tammara Massey; Leo Selavo; David Crawford; Bor-rong Chen; Konrad Lorincz; Victor Shnayder; Logan Hauenstein; Foad Dabiri; James Jeng; Arjun Chanmugam; David White; Majid Sarrafzadeh; Matt Welsh ; C.D. The Advanced Health and Disaster Aid Network: A Light-Weight Wireless Medical System for Triage IEEE Transactions on Biomedical Circuits and Systems, VOL. 1, NO. 32007.

[6] Shanshan Jiang; Yanchuan Cao; Sameer Iyengar; Philip Kuryloski; Roozbeh Jafari Yuan Xue; Ruzena Bajcsy; Stephen Wicker ; C.D. CareNet: An Integrated Wireless Sensor Networking Environment for Remote Healthcare . 
[7] A. Wood, G. Virone, T. Doan, Q. Cao, L. Selavo, Y. Wu, L. Fang, Z. He, S. Lin, J. Stankovic ; C.D. ALARM-NET: Wireless Sensor Networks for Assisted-Living and Residential Monitoring . Technical Report CS-2006-13, 2006.

[8] Chin-Feng Lai; Yueh-Min Huang; Jong Hyuk Park; Han-Chieh Chao; C.D. Adaptative Body Posture Analysis Using Collaborative Multi-Sensors for Elderly Falling Detection. IEEE Intelligent Systems , 2010.

[9] Philip Levis; Sam Madden; Joseph Polastre; Robert Szewczyk; Kamin Whitehouse; Alec Woo1; David Gay; Jason Hill; Matt Welsh; Eric Brewerl; David Culler; C.D. TinyOS: An Operating System for Sensor Networks.

[10] Alexandros Pantelopoulos; Nikolaos G. Bourbakis; C.D. A Survey on Wearable Sensor-Based Systems for Health Monitoring and Prognosis. IEEE Transactions on Systems, Man, and CyberneticsPart C: Applications and Reviews, Vol. 40, NO. 1, 2010.

[11] Bums, A. ; Greene, B. R.; McGrath, M. J.; O'Shea, T. J.; Kuris, B.; Ayer, S. M.; Strolescu, F.; Clonca, V.; C.D. SHIMMERTM - A Wireless Sensor Platform for Noninvasive Biomedical Research. IEEE Journals \& Magazines, VOL. 10, No. 9, 2010.

[12] Benott Latre; Bart Braem; Ingrid Moerman ; Chris Blondia; Piet Demeester ; C.D. A survey on wireless body area networks. Springer Science+Business Media, LLC , 2010.

[13] ZigBee specification ZB Alliance - ZigBee Document 053474r13, 2006.

[14] WLAN Interference to IEEE802.15.4. Available at: z-wavealliance.org, 2007.

[15] Min Chen; Sergio Gonzalez; Athanasios Vasilakos; Huasong Cao; Victor C. M. Leung ; C.D. Body Area Networks: A Survey. Springer Science+Business Media, LLC , 2010.

[16] Specification of the Bluetooth system SIG Bluetooth - Core, version, 2001.

[17] Pervez Khan; Md.Asdaque Hussain; Kyung Sup Kwak ; C.D. Medical Applications of Wireless Body Area Networks. IEEEInternational Journal of Digital Content Technology and its Applications Volume 3, Number 3, 2009.

[18] Ki-Dong Lee; Athanasios V. Vasilakos: C.D. Access stratum resource management for reliable uhealthcare service in LTE networks. Wireless Networks 17(7): 1667-1678, 2011.

[19] Gutierrez, R.; Fernandez, S.; Jesus Garcia, J.; Carlos Garcia, J.; Marnane, L.: C. D. Monitoring vital signs and location of patients by using ZigBee wireless sensor networks. Sensors, IEEE, 2011.

[20] Abderrahim Bourouis; Mohamed Feham,Abdelhamid Bouchachia: C. D. Ubiquitous Mobole Health Monitoring System For Elderly (UMHMSE). International Journal of Computer Science \& Information Technology (IJCSIT), Vol 3, No 3, June 2011. 